\title{
Hemodynamic and metabolic alterations associated with septic acute kidney injury in experimental sepsis
}

\author{
EH Post, F Su, K Hosokawa, FS Taccone, A Herpain, J Creteur, J -L Vincent, D De Backer \\ From ESICM LIVES 2015 \\ Berlin, Germany. 3-7 October 2015
}

\section{Introduction}

The role of renal perfusion in the development of septic acute kidney injury (AKI) remains elusive. When septic AKI develops in the presence of hypotension, renal dysfunction is considered to be caused by reduced renal blood flow and tissue hypoxia. However, an integrated view of the effects of sepsis on renal blood flow, oxygenation and local metabolism is currently lacking.

\section{Objectives}

To assess renal perfusion, kidney cortex metabolism and tissue oxygen tension in an ovine model of septic shock.

\section{Methods}

12 animals were randomized to sepsis $(n=8)$ or sham procedure $(n=4)$. A pre-calibrated flow probe was positioned around the renal artery to measure renal blood flow (RBF) and a catheter was inserted into the renal vein to measure renal vein oxygen content and calculate renal oxygen consumption, corrected for body surface area (renal $\left.\mathrm{VO}_{2} \mathrm{I}\right)$. A tissue oxygen-tension electrode and a microdialysis probe were inserted into the kidney cortex to measure interstitial oxygen tension $\left(\mathrm{tPO}_{2}\right)$ and glucose, lactate and pyruvate levels, respectively.

Sepsis was induced by injection of $1.5 \mathrm{~g} / \mathrm{kg}$ autologous feces into the abdominal cavity (TOh). Sham animals underwent similar surgery but no feces were injected. Treatment consisted of fluid-administration (Ringer's lactate and HES 130/4.2 in a 1:1 ratio) to keep pulmonary artery occluded wedge pressure at baseline levels.

The animals were observed for 18 hours and data were analyzed for main effect of time and interaction between group and time using linear mixed models. In case of significance, pairwise comparisons were carried out using Student's t-test. A p-value of less than 0.05 was considered statistically significant.

\section{Results}

The septic group developed renal dysfunction at $\mathrm{T} 12 \mathrm{~h}$, as evidenced by the occurrence of oliguria and a

Table 1. Renal hemodynamics and function

\begin{tabular}{|c|c|c|c|c|c|}
\hline & & TOh & T6h & $\mathrm{T} 12 \mathrm{~h}$ & $\mathrm{~T} 18 \mathrm{~h}$ \\
\hline \multirow[t]{2}{*}{$\mathrm{RBF}, \mathrm{mL} / \mathrm{min}$} & Sepsis & $165 \pm 32$ & $138 \pm 84$ & $71 \pm 38 \#_{,}^{*}$ & $45 \pm 23{\#,{ }^{*}}$ \\
\hline & Sham & $175 \pm 47$ & $185 \pm 47$ & $188 \pm 51$ & $198 \pm 61$ \\
\hline \multirow[t]{2}{*}{ Renal $\mathrm{VO}_{2} \mathrm{l}, \mathrm{mL} / \mathrm{min} / \mathrm{m}^{2}$} & Sepsis & $2.1 \pm 0.6$ & $1.9 \pm 0.7$ & $1.2 \pm 0.8 *$ & $0.9 \pm 0.5 \#_{,}^{*}$ \\
\hline & Sham & $1.6 \pm 0.6$ & $1.6 \pm 0.5$ & $1.7 \pm 0.4$ & $1.9 \pm 0.5$ \\
\hline \multirow[t]{2}{*}{ Creatinine Clearance, $\mathrm{mL} / \mathrm{min}$} & Sepsis & $77 \pm 25$ & $66 \pm 37$ & $12 \pm 0.6 \#^{*}{ }^{*}$ & $3 \pm 2 \#_{,}^{*}$ \\
\hline & Sham & $55 \pm 12$ & $53 \pm 24$ & $51 \pm 15$ & $52 \pm 27$ \\
\hline \multirow[t]{2}{*}{ UO, mL/kg/hour } & Sepsis & $2.2 \pm 1.1$ & $1.8 \pm 0.8$ & $0.3 \pm 0.2 \#,{ }^{*}$ & $0.1 \pm 0.1 \#_{,}^{*}$ \\
\hline & Sham & $1.5 \pm 0.8$ & $1.6 \pm 0.9$ & $1.6 \pm 0.8$ & $1.6 \pm 0.9$ \\
\hline
\end{tabular}


Table 2. Renal cortex metabolism

\begin{tabular}{lccccc}
\hline Glucose, $\mathrm{mg} / \mathrm{dL}$ & & T0h & T6h & T12h & T18h \\
\hline & Sepsis & $21 \pm 7$ & $20 \pm 12$ & $20 \pm 13$ & $16 \pm 17$ \\
\hline Lactate, $\mathrm{mmol} / \mathrm{L}$ & Sham & $28 \pm 14$ & $18 \pm 5$ & $19 \pm 5$ & $22 \pm 5$ \\
\hline Pyruvate, $\mu \mathrm{mol} / \mathrm{L}$ & Sepsis & $0.5 \pm 0.3$ & $0.7 \pm 0.3$ & $1.4 \pm 0.7 \#_{,}^{*}$ & $4.1 \pm 1.6 \#,{ }^{*}$ \\
\hline & Sham & $0.4 \pm 0.1$ & $0.7 \pm 0.3$ & $0.6 \pm 0.3$ & $0.4 \pm 0.2$ \\
\hline Tissue $\mathrm{PO}_{2}, \mathrm{mmHg}$ & Sepsis & $23 \pm 12$ & $44 \pm 12{ }^{*}$ & $69 \pm 33 \#,^{*}$ & $59 \pm 48$ \\
\hline & Sham & $29 \pm 9$ & $41 \pm 18$ & $38 \pm 18$ & $28 \pm 14$ \\
\hline
\end{tabular}

reduced creatinine clearance (table 1), concomitantly to the development of hypotension (MAP $47 \pm 15 \mathrm{mmHg}$ in control vs. $81 \pm 4 \mathrm{mmHg}$ in sham animals; $\mathrm{p}<0.05$ ). Low $\mathrm{RBF}$ and reduced renal $\mathrm{VO}_{2} \mathrm{I}$ were also observed after 12 hours. These findings were associated with increased cortical lactate and pyruvate levels and an elevated lactate pyruvate ratio (LPR) at T18h (table 2). In contrast, renal cortex $\mathrm{tPO}_{2}$ remained unchanged in both groups during the observation period.

\section{Conclusions}

In this model of experimental sepsis, severe AKI was associated with marked reduction in renal perfusion and metabolism although cortical $\mathrm{PO}_{2}$ was preserved.

Published: 1 October 2015

doi:10.1186/2197-425X-3-S1-A469

Cite this article as: Post et al:. Hemodynamic and metabolic alterations

associated with septic acute kidney injury in experimental sepsis.

Intensive Care Medicine Experimental 2015 3(Suppl 1):A469.

\section{Submit your manuscript to a SpringerOpen ${ }^{\circ}$ journal and benefit from:}

- Convenient online submission

- Rigorous peer review

- Immediate publication on acceptance

- Open access: articles freely available online

- High visibility within the field

- Retaining the copyright to your article

Submit your next manuscript at $>$ springeropen.com 